\title{
Konstruktive Potentiale einer Mikrostrukturgradierung von topologieoptimierten L-PBF-Bauteilen
}

\author{
Johannes Geis ${ }^{1 *}$, Holger Merschroth ${ }^{2}$, Tim Sommer ${ }^{1}$, Eckhard Kirchner ${ }^{1}$, \\ Matthias Weigold ${ }^{2}$ \\ ${ }^{1}$ Fachgebiet für Produktentwicklung und Maschinenelmente, TU Darmstadt \\ 2 Institut für Produktionsmanagement, Technologie und Werkzeugmaschinen, TU Darmstadt \\ * Korrespondierender Autor: \\ Johannes Geis \\ TU Darmstadt \\ Institute for Product Development and Machine Elements \\ Otto-Berndt-Straße 2, 64287 Darmstadt \\ Telefon: +49 6151/16-21186 \\ Mail: geis@pmd.tu-darmstadt.de
}

\begin{abstract}
The design space of topology optimizations is often limited by installation space limitations and interfaces to other components, which can result in local stress concentrations in the resulting notches. In this paper, the potential of microstructure grading was investigated by means of $\mathrm{FE}$ analyses on the basis of two components. In experimental investigations of L-PBF manufactured tensile specimens made of AISi10Mg, a producible variation range of the Young's modulus from 46 to 62 GPa could be determined. By grading the Young's modulus, a local stress reduction of $18.6 \%$ and $25 \%$ could be achieved by means of $\mathrm{FE}$ analysis, as well as a slight displacement of the stresses around the critical area.
\end{abstract}

\section{Keywords}

Additive Manufacturing, Laser Powder Bed Fusion, Microstructural Grading, Finite Element Analysis 


\section{Motivation und Einleitung}

Das pulverbettbasierte Laserstrahlschmelzen (L-PBF) gewinnt unter anderem aufgrund seiner hohen Gestaltungsfreiheiten in einigen Industriezweigen im Vergleich zu anderen Fertigungsverfahren immer mehr an Bedeutung. Die werkzeuglose Fertigung der Bauteile ermöglicht zudem frühe Prototypentests sowie schnelle Designiterationen [1]. Auch die geometrische Komplexität von Bauteilen spielt bei den Herstellungskosten eine untergeordnete Rolle, da diese maßgeblich von der Bauteilhöhe und dem zu belichtenden Bauteilvolumen abhängig sind. Gerade in Industriezweigen wie der Luft- und Raumfahrt, bei denen die Energieeffizienz der Produkte im Fokus steht, gewinnt daher die Fertigung geometrisch komplexer, leichtbauoptimierter Bauteile immer mehr an Bedeutung [2].

Durch die erhöhte Designfreiheit des L-PBF können ehemals konventionell gefertigte Baugruppen, die aus einer großen Anzahl an Bauteilen bestehen, zu einem bzw. wenigen Bauteilen zusammengefasst werden. Dadurch entfallen Materialdopplungen sowie Verbindungselemente, was sich wiederum in einem niedrigeren Gewicht und verkürzter Montagezeit niederschlägt. Um die Gewichtsreduktion einer Baugruppe zu maximieren, wird in der Praxis Topologieoptimierungssoftware verwendet, welche auf Basis von Bauteilschnittstellen und dem verfügbaren Bauraum automatisiert belastungsangepasste Geometrien errechnet. Bei einer Topologieoptimierung mit uneingeschränktem Optimierungsraum entsteht eine Bauteilgeometrie, welche unter den angenommenen äußeren Lasten einen nahezu homogenen Spannungszustand aufweist. Hierfür werden bei der numerischen Berechnung dieser Bauteile alle Volumenelemente entfernt, deren Spannung unter einem festgelegten Grenzwert liegt, um ein möglichst leichtbauoptimiertes Bauteil zu generieren. [3]

Die Bauteilkonsolidierung innerhalb eines Systems ist allerdings nur bis zu einem gewissen Grad möglich bzw. zielführend, da an lasttragenden Strukturen beispielsweise Sensoren, Aktoren oder austauschbare Verschleißteile montiert werden müssen. Zur Positionierung und Montage dieser Bauteile wird der Optimierungsraum der Topologieoptimierung daher oftmals eingeschränkt. Dies kann wiederum in lokalen Spannungsüberhöhungen in Kerben resultieren, die durch das Anstoßen der topologieoptimierten Geometrie an die Bauraumbeschränkungen innerhalb des Optimierungsraumes entstehen und somit die Belastbarkeit und Lebensdauer des Bauteils reduzieren. [4] Um dieser Problemstellung entgegenzutreten wird in diesem Beitrag untersucht, welche konstruktiven Potentiale sich aus der gezielten Prozessführung zur Steuerung der Abkühlraten während der Bauteilfertigung und somit mikrostruktureller Gradierung ergeben können. Hierfür sollen Spannungsüberhöhungen an kritischen Stellen abgebaut und in Bereiche verlagert werden, die weniger stark beansprucht sind (vgl. Abbildung 1) ohne die Makrogeometrie des Bauteils zu verändern.
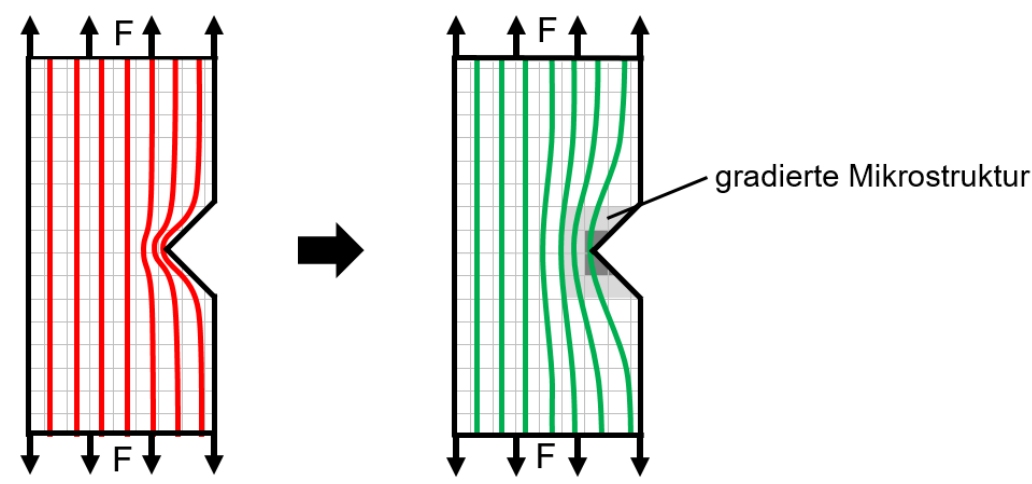

Abbildung 1: Prinzip der Spannungsumverteilung durch Gradierung der Mikrostruktur 


\section{Stand der Technik und Forschung}

Die Fertigung von Bauteilen mittels L-PBF wird zum aktuellen Stand der Technik mit statischen Prozessparametern durchgeführt. Unabhängig von der Belichtungsdauer und -intensität einer Schicht werden nachfolgende Schichten mit den gleichen Parametern gefertigt. Aufgrund eines zunehmenden Wärmeeintrags entlang der Aufbaurichtung des Bauteils führt dies zu einer anisotropen Mikrostruktur und dem Aufbau von Eigenspannungen. Dieser Effekt kann durch die Geometrie des zu fertigenden Bauteils, wie etwa einer größer werdenden Querschnittsfläche, verstärkt werden. [5, 6]

Mit dem L-PBF Verfahren können bei geschickter Prozessführung hohe Abkühlraten erreicht werden, die dazu führen, dass eine feine Gefügestruktur ausgebildet wird. Ebenso kann die Ausbildung eines groben Gefüges bei niedrigen Abkühlraten beobachtet werden; gerade vergleichsweise dünne Bauteilbereiche weisen aufgrund der geringen Wärmeableitung eine grobe Mikrostruktur auf. [7]

Daher wurde von Merschroth et. al ein Ansatz erarbeitet, mit dessen Hilfe die Abkühlrate eines Bauteils während der Fertigung durch dynamisch gesteuerte Prozessparameter konstant gehalten werden kann. Mit einer thermographischen Kamera wird der Bauraum während des Fertigungsprozesses überwacht und der Einfluss verschiedener Parameter auf die Bauteiltemperatur ermittelt. Dabei wird der Einfluss von den Belichtungsparametern, der Zeit zur Schichtgenerierung und den bauteilindividuellen geometrischen Parametern auf die Bauteiltemperatur berücksichtigt. Mit den aufgezeichneten Überwachungsdaten wird ein neuronales Netz trainiert, welches zuverlässig die Temperaturentwicklung innerhalb eines Bauteils vorhersagen kann (vgl. Abbildung 2a). Im zweiten Schritt wird ein umgekehrtes neuronales Netz verwendet, um die Laserleistung zum Erreichen der maximal zulässigen Temperatur zu ermitteln. Der Fertigungsanlage wird nun wird eine Prozessparameter-Matrix zugeführt, bei der die Laserleistung schichtweise für alle Voxel innerhalb festgelegter Grenzwerte zugewiesen ist. Die Bauteiltemperatur kann mit dieser Steuerung effektiv unter dem angestrebten Grenzwert gehalten werden (vgl. Abbildung 2b) und somit der Ausbildung einer mikrostrukturellen Anisotropie effektiv entgegenwirken. [8]

a)
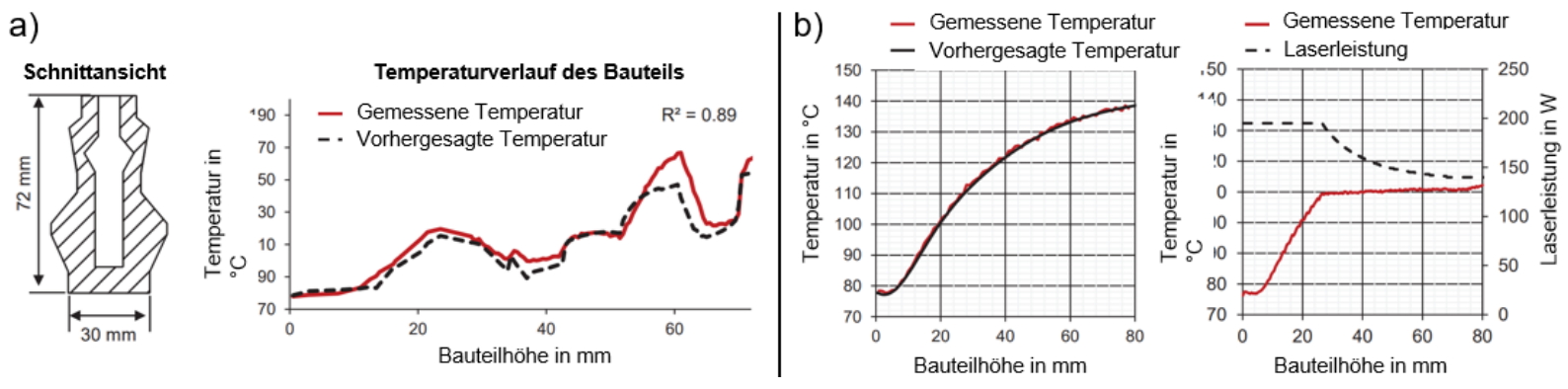

Abbildung 2: a) Vergleich von gemessener und vorhergesagter Bauteiltemperatur (rechts) einer komplexen Geometrie (links); b) Temperaturverlauf für einen unangepassten Prozess (links) und den angepassten Prozess (rechts) [Merschroth et al. 2021]

Auch eine gezielt eingestellte Anisotropie der Mikrostruktur konnte durch die Variation der Belichtungsstrategie sowie -parameter bei der Fertigung einer Turbinenschaufel aus IN718 bereits exemplarisch umgesetzt werden. Attard et al. haben dazu die herstellbaren Mikrostrukturen analysiert, um Rückschlüsse auf den Einfluss der Prozessparameter auf die Abkühlraten abschätzen zu können. Anschließend wurde ein thermisches Modell aufgesetzt, um die vorübergehende Bauteiltemperatur während des Fertigungsprozesses als Eingangsgröße für ein numerisches Kornwachstumsmodell abschätzen zu können. Mit Hilfe dieser Informationen konnte eine Belichtungsstrategie für die Herstellung einer gradierten 
Mikrostruktur in einer Turbinenschaufel entwickelt und erfolgreich angewendet werden (vgl. Abbildung 3). [9]
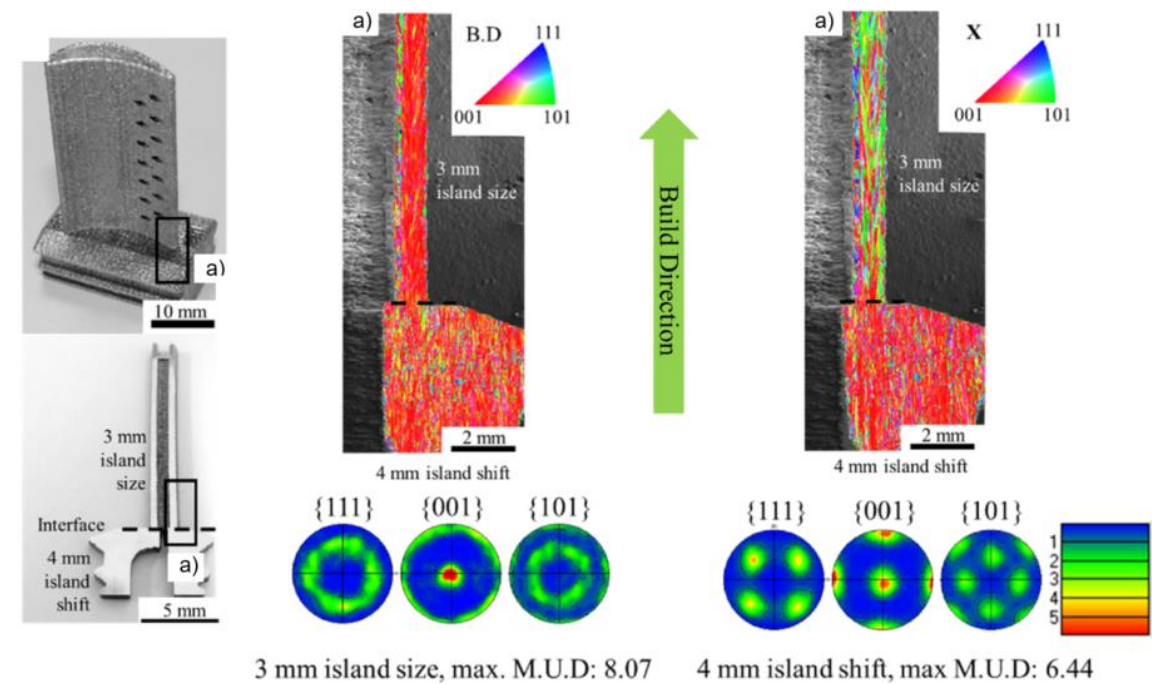

Abbildung 3: Inverse Polkarte der Schnittansicht entlang Aufbau- und Querrichtung der „GRADEDBLADE“ sowie Polfiguren der beiden Bauteilbereiche mit unterschiedlichen Belichtungsstrategien [Attard et al., 2020]

Zur numerischen Berechnung von topologieoptimierten Strukturen existieren unterschiedliche Algorithmen. Alle haben allerdings gemein, dass zunächst die Werkstoffeigenschaften, der verfügbare Optimierungsraum, die notwendigen Anschlussgeometrien sowie die Betriebslastfälle definiert werden müssen. Darüber hinaus können verfahrensbedingte Fertigungsrestriktionen sowie Optimierungsziele, wie bspw. eine hohe Steifigkeit oder eine maximale Massenreduktion, festgelegt werden. Einer der gängigsten Algorithmen zur Topologieoptimierung berechnet in der anschließenden FEAnalyse (Finite Elemente Analyse) den vorliegenden Spannungszustand aller Volumenelemente im Optimierungsraum. Nachfolgend werden alle Volumenelemente entfernt, die weit unterhalb ihrer zulässigen Spannung beansprucht werden. Das entstandene, reduzierte FE-Modell wird nun geglättet und in ein CAD-Modell überführt. [3]

Das CAD-Modell wird daraufhin erneut mittels einer FE-Analyse und den zugrundeliegenden Betriebslastfällen auf Anforderungserfüllung überprüft und gegebenenfalls noch einmal formoptimiert. Dabei werden vor allem Spannungsspitzen durch Anpassung der Makrogeometrie reduziert, um die Betriebsfestigkeit des Bauteils sicherzustellen [10]. Dies ist allerdings nur in Bauteilbereichen möglich, die den Optimierungsraum nicht vollständig ausnutzen. Bei Bauteilbereichen, die an die Bauraumbegrenzung anstoßen kann trotz Topologieoptimierung eine inhomogene Spannungsverteilung im Bauteil vorliegen, die abhängig von den Materialkennwerten und den äußeren Lasten zum Versagen führen kann [3].

\section{Problemstellung und Forschungsfrage}

Durch die Möglichkeiten der Steuerung von Abkühlraten sowie Anpassung der Belichtungsstrategie während des Fertigungsprozesses werden in Zukunft auch die „as-printed“ Bauteileigenschaften verbessert. Dies wird das Anwendungsspektrum von L-PBF gefertigten Bauteilen erweitern, da je nach Anwendungsfall keine Wärmenachbehandlung zur Steigerung der mechanischen Eigenschaften mehr notwendig ist. Die Technologie neuronaler Netze könnte in Kombination mit einer angepassten Belichtungsstrategie zur gezielten Gradierung der Abkühlgeschwindigkeit verwendet werden, was wiederum eine Gradierung der 
Mikrostruktur ermöglicht. Dadurch können Spannungsspitzen an kritischen Stellen reduziert und die Lebensdauer sowie maximale Belastbarkeit erhöht werden. Ziel des hier vorgestellten Ansatzes ist es daher, mittels FE-Analyse zu überprüfen, ob eine prozessgetriebene Gradierung der Materialeigenschaften einen nennenswerten Mehrwert zur bisherigen Fertigung lasttragender Strukturen beitragen kann.

\section{4. Überprüfung der konstruktiven Potentiale einer Mikrostrukturgradierung}

Die Spannungsverlagerung innerhalb des Bauteils kann über eine Gradierung der Steifigkeit umgesetzt werden, welche wiederum von der makroskopischen Geometrie und den Werkstoffeigenschaften abhängig ist [11]. Da dieser Ansatz eine Beibehaltung der Makrogeometrie zum Ziel hat, muss ein auslegungsrelevanter Werkstoffkennwert festgelegt werden.

In der Regel werden Bauteile unterhalb der 0,2\%-Dehngrenze ausgelegt, um plastische makroskopische Verformungen zu vermeiden und eine dauerhafte Belastung durch die im Betrieb auftretenden Lasten zu gewährleisten. Eine kontrollierte plastische Verformung in geringem Maße ist an kritischen Stellen mit Spannungsüberhöhung jedoch bei duktilen Werkstoffen toleriert, da die Bauteile ansonsten wesentlich massiver ausgelegt werden müssten [12]. Mit einer Erhöhung des Elastizitätsmoduls in den übrigen Bereichen wird die Spannungsüberhöhung abgebaut und ins Innere des Bauteils verlagert. Dieser Effekt verstärkt sich durch die Erhöhung der Nachgiebigkeit in den kritischen Stellen. Der Spannungszustand eines Bauteilbereiches mit unterschiedlichen Elastizitätsmoduln kann analog zur Parallelschaltung zweier Spiralfedern mit unterschiedlichen Federkonstanten betrachtet werden. Die steifere Feder bzw. der Bauteilbereich mit höherem Elastizitätsmodul nimmt bei gleicher Längenänderung einen größeren Teil der anliegenden Spannung auf. Diese Verlagerung trägt auch dazu bei, dass sich eine geringere plastische Verformung an kritischen Bereichen einstellt. Daher wird der Elastizitätsmodul als Optimierungsgröße ausgewählt.

\subsection{Experimentelle Ermittlung der herstellbaren Werkstoffkennwerte}

Als Grundlage für eine Untersuchung der konstruktiven Potentiale einer Mikrostrukturgradierung mittels FE-Analyse, muss zunächst geprüft werden, in welchem Bereich die Werkstoffeigenschaften durch die Prozessparameter beeinflusst werden können. Hierzu werden Zugproben auf einer EOS M290 mit dem Werkstoff AISi10Mg hergestellt. Auf der Basis von Voruntersuchungen werden die Hauptprozessparameter Laserleistung, Scangeschwindigkeit sowie Spurabstand variiert und deren Auswirkung auf das Elastizitätsmodul untersucht. Die Bauteile werden mittels Streifenbelichtung mit einer Streifenbreite von $5 \mathrm{~mm}$ und einer Streifenüberlappung von $100 \mu \mathrm{m}$ gefertigt. Dabei werden ausschließlich Prozessparameter zur Fertigung von Zugproben verwendet, mit denen Bauteile mit einer relativen Dichte $>99,5 \%$ gefertigt werden können, um den Einfluss prozessindizierter Poren auf die mechanischen Eigenschaften zu minimieren. Auch die verfahrensbedingte hohe Oberflächenrauheit der Zugproben hat einen Einfluss auf die mechanische Prüfung der Werkstoffeigenschaften und muss daher spanend nachbearbeitet werden [13]. Die Probenform wird nach DIN 50125 ausgewählt und auf die Form E 5 × 10 × 40 festgelegt.

\subsection{Vorgehensweise zur Mikrostrukturauslegung mittels FEM}

Zur Überprüfung des konstruktiven Potentials wird ein Bauteil mittels Finite Elemente Simulation analysiert und auf Grundlage dieser Ergebnisse gradiert. Der Ablauf des Optimierungstools ist in Abbildung 4 schematisch dargestellt. Nach Definition des Bereiches, in der das Elastizitätsmodul variiert werden kann, wird die Bauteilgeometrie in gleichgroße Voxel eingeteilt. Diese Voxel dienen im Verlauf der Optimierung dazu, den unterschiedlich 
großen finiten Elementen innerhalb dieser Voxel zeitgleich neue Elastizitätsmodule zuweisen zu können. Nach Angabe der äußeren Lasten sowie vorhandener Randbedingungen wird der Spannungszustand des Bauteils mit homogenen Werkstoffkennwerten berechnet und die Gittergröße an hoch beanspruchten Stellen iterativ verfeinert. Mittels Gaußpunkte werden anschließend die Durchschnittsspannungen in den Voxeln bestimmt, auf deren Basis die anschließende Gradierung stattfindet. Der Elastizitätsmodul dieser Voxel wird in aufeinanderfolgenden FE-Analysen nach folgendem Konzept in linear verteilten, diskreten Schritten iterativ an den vorliegenden Spannungszustand angepasst: Das auftretende Spannungsspektrum wird zunächst in zwei Bereiche unterteilt. Die Voxel, welche sich im unteren Bereich des Spannungsspektrums befinden, werden mit dem höchsten Elastizitätsmodul versehen, um eine möglichst steife Struktur zu gewährleisten. Den übrigen Voxeln wird entsprechend der vorliegenden Spannungshöhe in linearer Abstufung ein Elastizitätsmodul im herstellbaren Variationsbereich zugewiesen. Voxel mit hoher Spannung werden mit einem niedrigen Elastizitätsmodul belegt und vice versa. Wenn die auftretenden Spannungen durch Anpassung des Elastizitätsmoduls nicht weiter reduziert werden können, ist das Abbruchkriterium erfüllt und die Optimierung wird beendet.

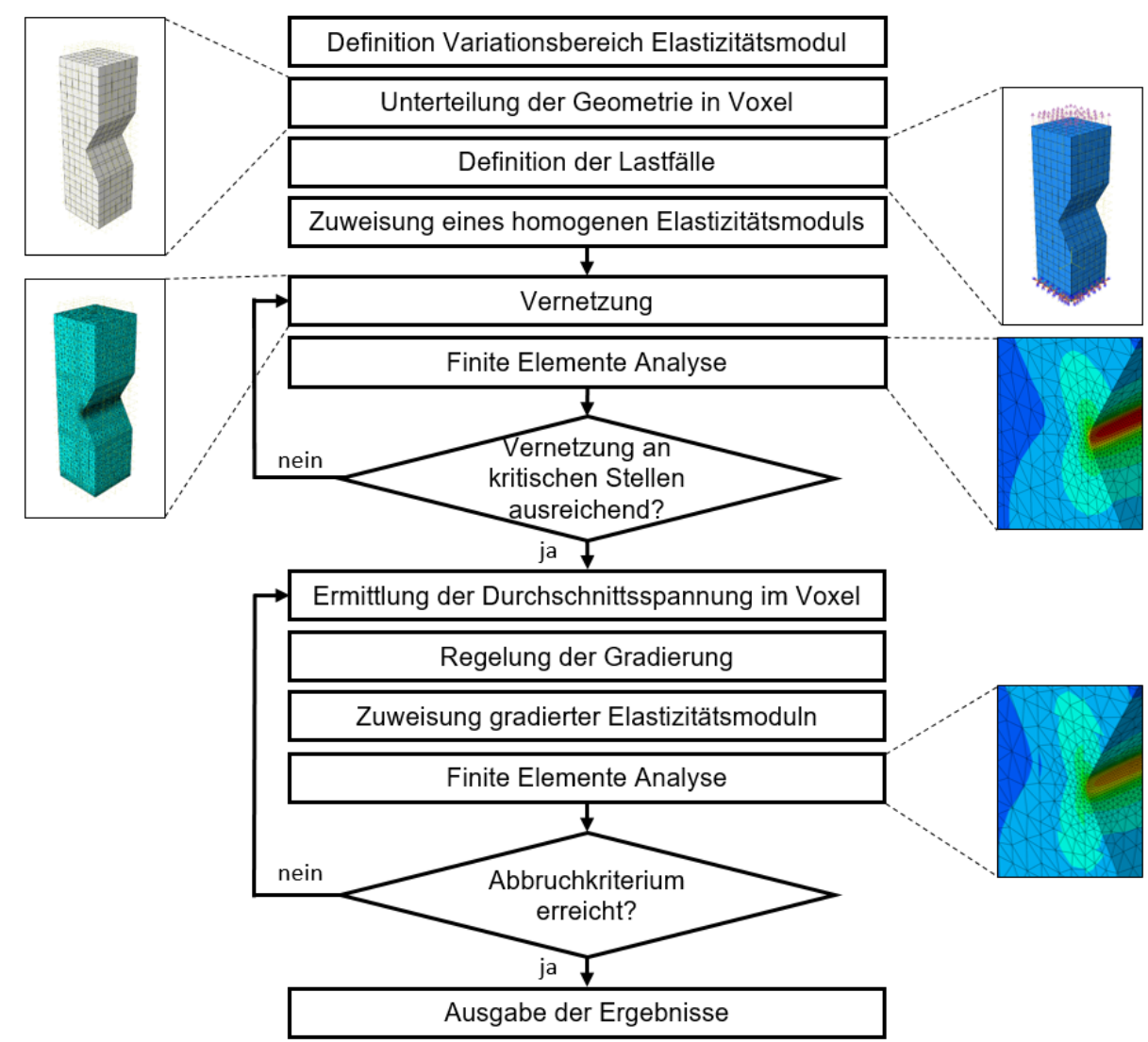

Abbildung 4: Schematischer Aufbau des Tools zur Mikrostrukturauslegung

\section{Ergebnisse und Diskussion}

Durch eine Variation der Hauptprozessparameter Laserleistung, Scangeschwindigkeit und Spurabstand kann ein signifikanter Einfluss auf die resultierenden mechanischen Eigenschaften erzielt werden. Folgend wird die Linienenergiedichte als Referenzgröße definiert, um valide Vergleiche zwischen Prozessparameterkombinationen zu ziehen.

Der Elastizitätsmodul kann, unter der Voraussetzung einer Mindestdichte von 99,5\%, zwischen 46 und $62 \mathrm{GPa}$ variiert werden. Es besteht eine Korrelation des Elastizitätsmoduls zu den Prozessparametern, so dass gezielt zwischen zwei Bereichen unterschieden werden kann. Für Linienenergiedichten kleiner $0,25 \mathrm{~J} / \mathrm{mm}^{2}$ ergeben sich Werte für den 
Elastizitätsmodul zwischen 55 und $62 \mathrm{GPa}$ (vgl. Abbildung 5). Für Linienenergiedichten größer $0,5 \mathrm{~J} / \mathrm{mm}^{2}$ ergeben sich Werte von 46 bis $52 \mathrm{GPa}$ für den Elastizitätsmodul. Es ist keine signifikante Abhängigkeit des Elastizitätsmoduls zum Spurabstand erkennbar. Die Standardabweichung des Elastizitätsmoduls je untersuchtem Prozessparametersatz beträgt bei einer Probenzahl von 3 zwischen 6 und $12 \mathrm{GPa}$ und ist durch Fehlerbalken dargestellt.

Das höhere Elastizitätsmodul für Linienenergiedichten kleiner $0,25 \mathrm{~J} / \mathrm{mm}^{2}$ resultiert aus den typischerweise höheren Abkühlgeschwindigkeiten kleiner Schmelzbäder und den damit einhergehenden feineren Gefügestrukturen. Die feinen Gefügestrukturen bewirken einen höheren Versetzungswiderstand an den Korngrenzen und somit höhere Festigkeitseigenschaften. Eine Betrachtung der Ausscheidungen, Kornwachstumsrichtung oder der Korngröße zur Quantifizierung der Mikrostruktur wurde nicht durchgeführt. Linienenergiedichten größer $0,5 \mathrm{~J} / \mathrm{mm}^{2}$ bewirken hingegen ausgeprägte Schmelzbäder und größere thermische Einflussbereiche mit folgend niedrigeren Temperaturgradienten und niedrigeren Abkühlgeschwindigkeiten. Durch die längeren Abkühlzeiten steigt die Korngröße und die Anzahl der Korngrenzen wird reduziert.

Zur Quantifizierung der Korrelationen zwischen Elastizitätsmodul und Prozessparametern sind weitere experimentelle Untersuchungen notwendig. Grundsätzlich ermöglicht die Variation der Prozessparameter eine Einstellbarkeit des Elastizitätsmoduls zwischen 46 und $62 \mathrm{GPa}$. Diese Variationsfreiheit durch gezielte Wahl der Prozessparameter dient als Grundlage für die folgend durchgeführte numerische Potentialuntersuchung des Mikrostrukturdesigns.

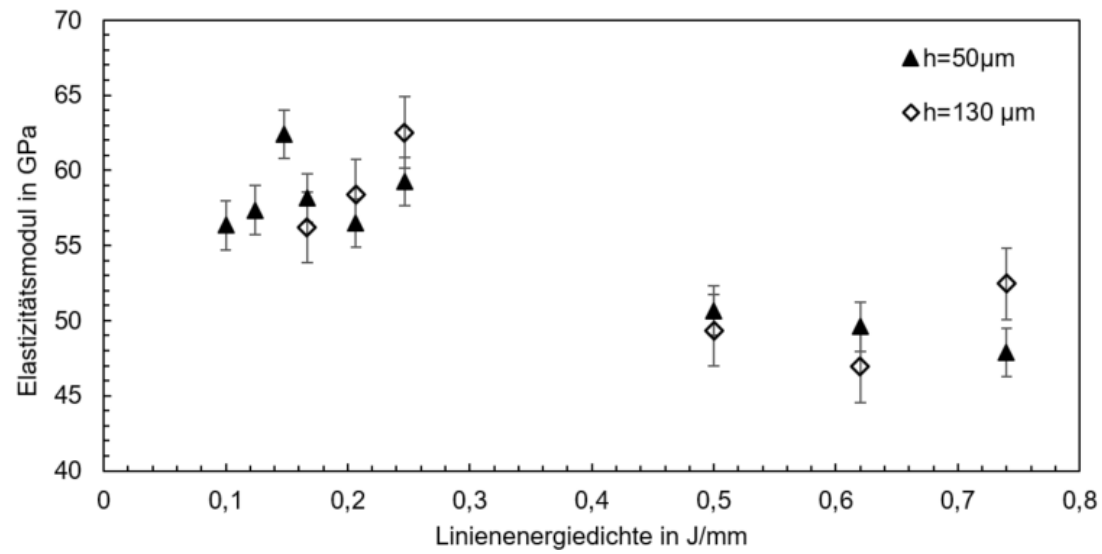

Abbildung 5: Darstellung des Elastizitätsmoduls in Abhängigkeit der Linienenergiedichte für unterschiedliche Spurabstände

Basierend auf dem ermittelten Variationsbereich für das Elastizitätsmodul wird zunächst eine gekerbte Probe unter einachsiger Zugbelastung mit dem in 4.2 vorgestellten Tool untersucht, um die Vorgehensweise des Tools zur Mikrostrukturauslegung zu überprüfen. Die Probengeometrie hat die Dimensionen von $30 \times 30 \times 100 \mathrm{~mm}$ und wird mit einer ausgerundeten Kerbe auf einer Seite des Bauteils versehen. Das Bauteil ist in $6 \times 6 \times 20$ Voxel unterteilt, die für die erste FE-Analyse mit einem Elastizitätsmodul von $62 \mathrm{GPa}$ belegt werden. Die Probe ist auf der Unterseite fest eingespannt und wird an der Oberseite durch eine Kraft von $3 \mathrm{~N}$ auf Zug belastet. Die Vernetzung des Gitters im Bereich der Kerbe wurde durch das Optimierungstool im Vergleich zu den umliegenden Bereichen verfeinert, wodurch eine genauere Berechnung der maximal auftretenden Spannung möglich wird.

Mit einem homogenen Elastizitätsmodul von $62 \mathrm{GPa}$ stellt sich eine maximale Spannung von $248 \mathrm{MPa}$ im Kerbgrund ein (vgl. Abbildung 6a). Nach vier Iterationsschritten der Gradierung wird das Abbruchkriterium erreicht und die Spannung auf $202 \mathrm{MPa}$ gesenkt, was einer Reduzierung um 18,6\% entspricht (vgl. Abbildung 6b). Neben der Senkung der maximalen Spannung in der Kerbe ist eine geringfügige Verlagerung der Spannung in den angrenzenden 
Bereichen erkennbar. Dies zeigt sich an den markierten Stellen 1, 2, 3 sowie 4 und ist durch unterschiedliche Elastizitätsmoduln in aneinander angrenzenden Voxeln erklärbar. Die örtlich näher zur Kerbe gelegenen Voxel nehmen aufgrund des reduzierten Elastizitätsmoduls eine geringere Spannung auf, wohingegen weiter entfernte Voxel dies mit einer etwas erhöhten Spannung ausgleichen. Im Bereich der Kerbe hat das Optimierungstool erwartungsgemäß ein Elastizitätsmodul von $46 \mathrm{GPa}$ zugewiesen und diesen mit zunehmender Entfernung in 5 äquidistanten Abstufungen bis auf $62 \mathrm{GPa}$ erhöht.

a)

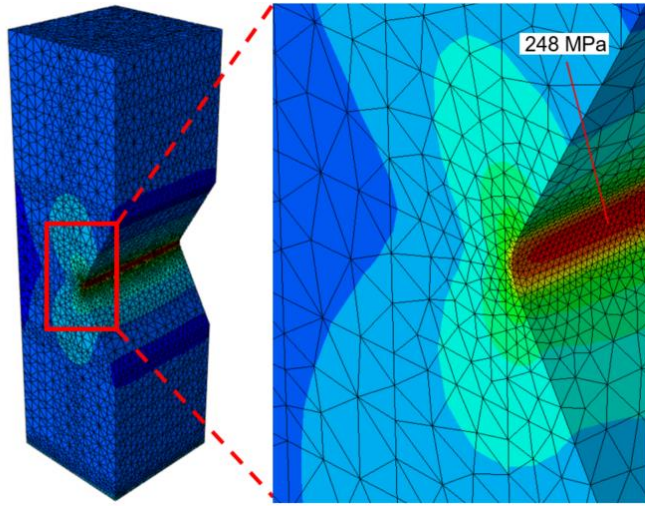

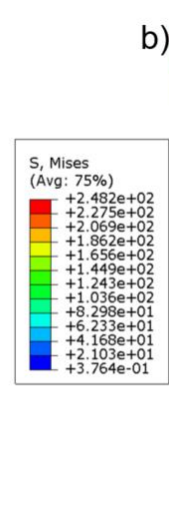
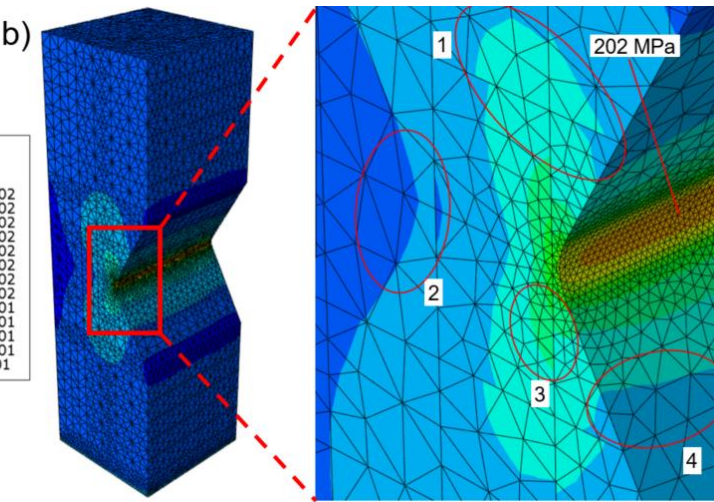

Abbildung 6: a) FE-Analyse des gekerbten Bauteils mit homogenem b) und gradiertem Elastizitätsmodul

Nach Plausibilisierung der Vorgehensweise des Optimierungstools wird nun eine topologieoptimierte Struktur (vgl. Abbildung 7) untersucht. Das Bauteil ist an der Bodenplatte fest eingespannt und wird an den beiden oberen Platten mit jeweils einer Flächenlast in Plattenebene beaufschlagt. In der Mitte zwischen den beiden Platten befindet sich eine Bauraumbeschränkung, die dazu führt, dass bei der Topologieoptimierung kein Material in diesem Bereich angesiedelt werden kann und das Modell an die Bauraumbeschränkung stößt. Im Vergleich zwischen Abbildung 7a und b ist zu erkennen, dass die Oberfläche sowie der Übergang von der Ausgangs- zur numerisch berechneten Geometrie geglättet und abgerundet wurden. Dies reduziert scharfe Geometrieübergänge und beugt damit effektiv Spannungsüberhöhungen vor. Im Bereich der Bauraumbeschränkung ist eine solche Verrundung nicht zulässig weswegen das Bauteil ideal geeignet ist, um die etwaig vorliegende Spannungsüberhöhung mittels Mikrostrukturgradierung zu reduzieren.

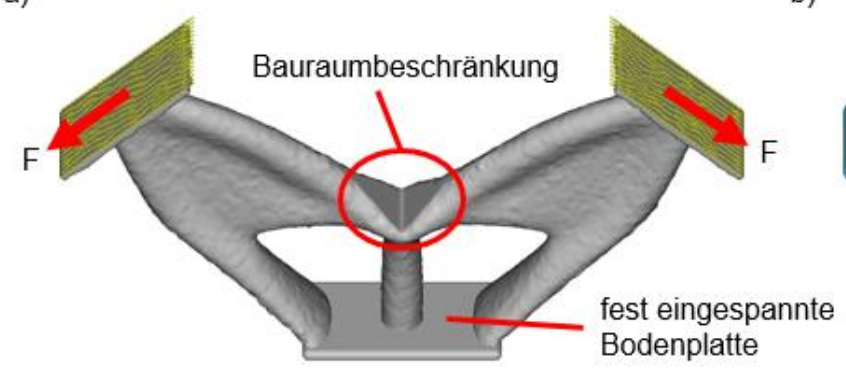

b)

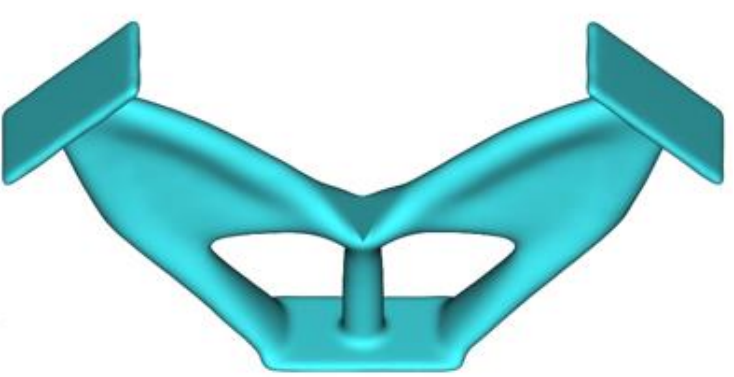

Abbildung 7: a) FE-Modell und b) geglättetes CAD-Modell des topologieoptimierten Bauteils

Das topologieoptimerte Bauteil besitzt die Abmessungen $62 \times 110 \times 16 \mathrm{~mm}$ und ist in $31 \times 55 \times 8$ Voxel unterteilt; entlang der gerundeten Kerbe befinden sich 8 Voxel. In Abbildung 8 ist eine Schnittansicht der FE-Analyse dargestellt, um auch den Spannungszustand im Inneren des Bauteils sichtbar zu machen. Mit einem homogenen Elastizitätsmodul zeigt die FE-Analyse eine maximale Spannung von $190 \mathrm{MPa}$ im Kerbgrund. Mit Hilfe des Optimierungstools konnte diese Spannung um ca. 25\% auf $142 \mathrm{MPa}$ reduziert 
werden. Wie bereits bei der gekerbten Zugprobe wird im Bereich der Kerbe das geringste Elastizitätsmodul von $46 \mathrm{GPa}$ zugewiesen. Unterhalb der Kerbe nimmt das Elastizitätsmodul auf $50 \mathrm{GPa}$ zu und erhöht sich in 3 weiteren Abstufungen auf $62 \mathrm{GPa}$. In Abbildung 8b ist erkennbar, dass die Spannung in den Bereichen 2, 3 und 4 effektiv verringert bzw. umverlagert werden konnte. An den Voxelgrenzen sind die Spannungssprünge im Vergleich zur gekerbten Zugprobe deutlicher zu erkennen, was auf etwas größere Sprünge im Elastizitätsmodul zurückzuführen ist. In Bereich 1 tritt die maximale Spannung auf, welche deutlich reduziert sowie das in grün/türkis gefärbte direkte Umfeld der Kerbe verkleinert werden konnte.

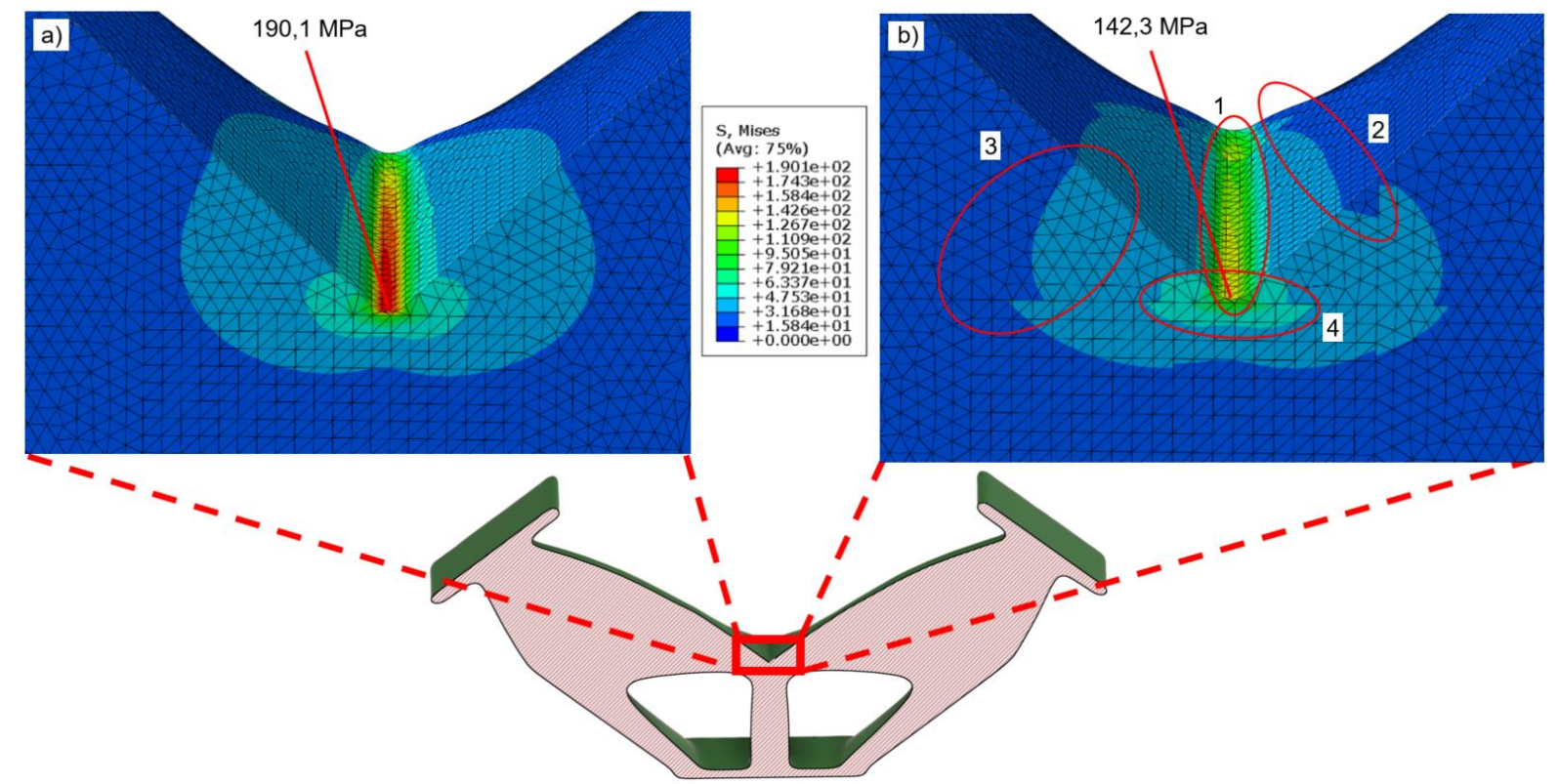

Abbildung 8: Schnittansicht einer FE-Analyse im Bereich der kritischen Stelle des topologieoptimierten Bauteils mit a) homogenem und b) gradiertem Elastizitätsmodul

Die auftretenden Spannungssprünge an den Voxelgrenzen wirken prinzipiell wie Kerben innerhalb des Bauteils. In der Realität wären solche klaren Abgrenzungen der Materialbeschaffenheit jedoch nicht herstellbar, da anzunehmen ist, dass die Wärmeeinflusszone benachbarter Voxel einen fließenden Übergang zwischen den unterschiedlichen Gefügen bewirkt. Dies ist auch der Grund für die Wahl von verhältnismäßig großen Voxeln, da zum aktuellen Zeitpunkt nicht abschätzbar ist, inwiefern eine Materialgradierung auf solch kleinem Raum durch die Steuerung der Abkühlraten möglich ist. Bei FE-Analysen mit wesentlich kleineren Voxeln konnte durch eine feinere Abstufung der Elastizitätsmoduln größere Spannungsreduzierungen und -verlagerungen erzielt werden, allerdings wurde dies als tendenziell nicht bzw. schwer realisierbar eingeschätzt. Um eine konservative Einschätzung der Potentiale einer Mikrostrukturgradierung erlangen zu können, wurde daher mit großen Voxeln simuliert.

Des Weiteren ist das Potential der Spannungsreduzierung maßgeblich vom vorliegenden Belastungsfall und der untersuchten Geometrie abhängig. Bei der Gradierung weiterer Geometrien konnte festgestellt werden, dass die theoretisch erreichbare Spannungsreduktion teilweise auch geringer ausfallen kann. Dies macht eine bauteilindividuelle Einzelfallbetrachtung des Potentials zur Spannungsreduzierung notwendig. Bei den gezeigten Probekörpern konnte eine erhebliche Reduktion der maximal auftretenden Spannung von 18 bzw. 25\% erreicht werden. Dies verspricht Potential zur Lebensdauererhöhung von topologieoptimierten Bauteilen, da gerade diese häufig keinen Raum zur geometrischen Anpassung kritischer Bauteilbereiche bieten. Eine weitere Untersuchung der hier vorgestellten Vorgehensweise ist daher anzustreben. 


\section{Zusammenfassung und Ausblick}

Im vorliegenden Beitrag wurden die konstruktiven Potentiale einer Mikrostrukturgradierung exemplarisch anhand zweier Bauteile mittels FE-Analysen überprüft. In experimentellen Untersuchungen von L-PBF gefertigten Zugproben aus AISi10Mg konnte ein herstellbarer Variationsbereich des Elastizitätsmoduls von 46 bis $62 \mathrm{GPa}$ ermittelt werden. Durch die Gradierung des Elastizitätsmoduls konnte mittels FE-Analyse eine lokale Spannungsreduktion von $18,6 \%$ bzw. $25 \%$ erzielt sowie eine Verlagerung der Spannungen erzielt werden. Das Potential zur Spannungsreduktion ist dabei maßgeblich von der vorliegenden Bauteil- und Kerbgeometrie sowie der Größe der lokalen Spannung abhängig. Insbesondere für Bauteile, die aufgrund von Bauraumrestriktionen nicht in ihrer Geometrie veränderbar sind, bietet der vorgestellte Ansatz Potential zur Erhöhung der Lebensdauer unter statischer und vor allem dynamischer Belastung.

Basierend auf den oben genannten Ergebnissen sind weitergehende Untersuchungen durchzuführen. Zunächst müssen gekerbte Zugproben mit gradierter Mikrostruktur hergestellt und mit nicht-gradierten verglichen werden. Mit Hilfe dieser Ergebnisse kann anschließend die vorgestellte Vorgehensweise zur Mikrostrukturauslegung validiert und gegebenenfalls angepasst werden - gerade die Wahl der Voxelgröße sollte dabei näher untersucht werden. Die Fertigung komplexer Geometrien wird in Zukunft eine engere Kopplung von Design und Prozessparametern notwendig machen, um Abkühlraten und Erstarrungsverhalten gezielt beeinflussen und ungewollte prozessindizierte Gefügegradierungen vermeiden zu können. Auch weitere konstruktive Features wie z.B. eine Härtung der Randbereiche oder das Einbringen von Sollbruchstellen können über die Kopplung von Design und Prozessparametern umgesetzt werden.

\section{Literaturverzeichnis}

[1] Reichwein, J.; Vogel, S.; Schork, S.; Kirchner, E.: On the Applicability of Agile Development Methods to Design for Additive Manufacturing. In: Procedia CIRP 91 (2020), S. 653-658.

[2] Zhu, J. et al.: A review of topology optimization for additive manufacturing: Status and challenges. In: Chinese Journal of Aeronautics (2021), Nr. 34, S. 91-110.

[3] Walzl, A.; Buchmayr, B.: Topologieoptimierung - Entwicklungswerkzeug für die additive Fertigung. In: BHM Berg- und Hüttenmännische Monatshefte 162 (2017), Nr. 3, S. 110-116.

[4] Saleem, W.; Yuqing, F.: Strategy for optimal configuration design of existing structures by topology and shape optimization tools. World Academy of Science, Engineering and Technology 37. (2010), S. 1058-1066.

[5] Brandl, E. et al.: Additive manufactured AISi10Mg samples using Selective Laser Melting (SLM): Microstructure, high cycle fatigue, and fracture behavior. In: Materials \& Design 34. (2012), S. 159-169.

[6] Würtenberger, J.; Reichwein, J.; Kirchner, E.: Using the Potentials of Additive Manufacturing by a systematic Linkage of the Manufacturing Process to Product Design. In: Proceedings of the 15th International Design Conference. Dubrovnik, 2018, S. 1465-1476.

[7] Oliveira, J. P.; Santos, T. G.; Miranda, R. M.: Revisiting fundamental welding concepts to improve additive manufacturing: From theory to practice. In: Progress in Materials Science 107 (2020), Nr. 100590.

[8] Merschroth, H.; Kniepkamp, M.; Weigold, M.: Predicting and controlling the thermal part history in powder bed fusion using neural networks. In: Bourell, D.; Beaman, J.; Crawford, R. (Hrsg.): Proceedings of the 30th Annual International Solid Freeform Fabrication Symposium. Austin, USA: (2021), S. 161-171.

[9] Attard, B. et al.: Microstructural control during laser powder fusion to create graded microstructure Nisuperalloy components. In: Additive Manufacturing 36 (2020), Nr. 101432.

[10] Mattheck, C.; Bethge, K.: Zur Plausibilität der Methode der Zugdreiecke. In: Materialwissenschaft und Werkstofftechnik 36 (2005), Nr. 11, S. 748-749.

[11] Lippert, R.; Lachmayer, R.: Validierung laserstrahlgeschmolzener Strukturbauteile aus AISi10Mg. In: Lachmayer, R.; Lippert, R.; Kaierle, S. (Hrsg.): Additive Serienfertigung: Erfolgsfaktoren und Handlungsfelder für die Anwendung. Berlin, Heidelberg: Springer, 2018, S. 39-52.

[12] Bürgel, R.: Festigkeitslehre und Werkstoffmechanik. Wiesbaden: Vieweg+Teubner Verlag, 2005. [13] Hankele, M; Werz, M.; Weihe, S.: Quantitative Untersuchungen der statischen und zyklischen Festigkeitseigenschaften von additiv gefertigten Proben aus AISi10Mg. In: Richard, H.; Schramm, B.; Zipsner T. (Hrsg.): Additive Fertigung von Bauteilen und Strukturen. Springer Vieweg, Wiesbaden, 2019, S. 127-144. 